\title{
Las elecciones presidenciales de 1989 en Brasil
}

Victor Manuel Durand Ponte

$\mathrm{E}$

sta ponencia trata de analizar el significado de las elecciones presidenciales realizadas en noviembre (primer turno) y en diciembre (segundo turno) del año pasado, desde el punto de vista de la transición política que se vive en Brasil, de especial significación para el sistema de partidos políticos. En virtud de que se presenta ante un público no brasileño y lo mismo sucederá con la publicación, se hace necesario dar algunos antecedentes generales para hacer comprensible el último proceso electoral.

\section{ANTECEDENTES}

Cabe resaltar que desde 1960 no se habían llevado a cabo comicios para elegir presidente de la república en Brasil. En aquel año, el electorado brasileño llevó a Janio Quadros a la primera magistratura, quien renunció a su cargo en 1962 dando lugar a que João Goulart, vicepresidente electo, ocupara la presidencia. En marzo de 1964, este presidente fue depuesto por el golpe militar, dando inicio a la dictadura militar que se extendió hasta 1986 cuando un civil 


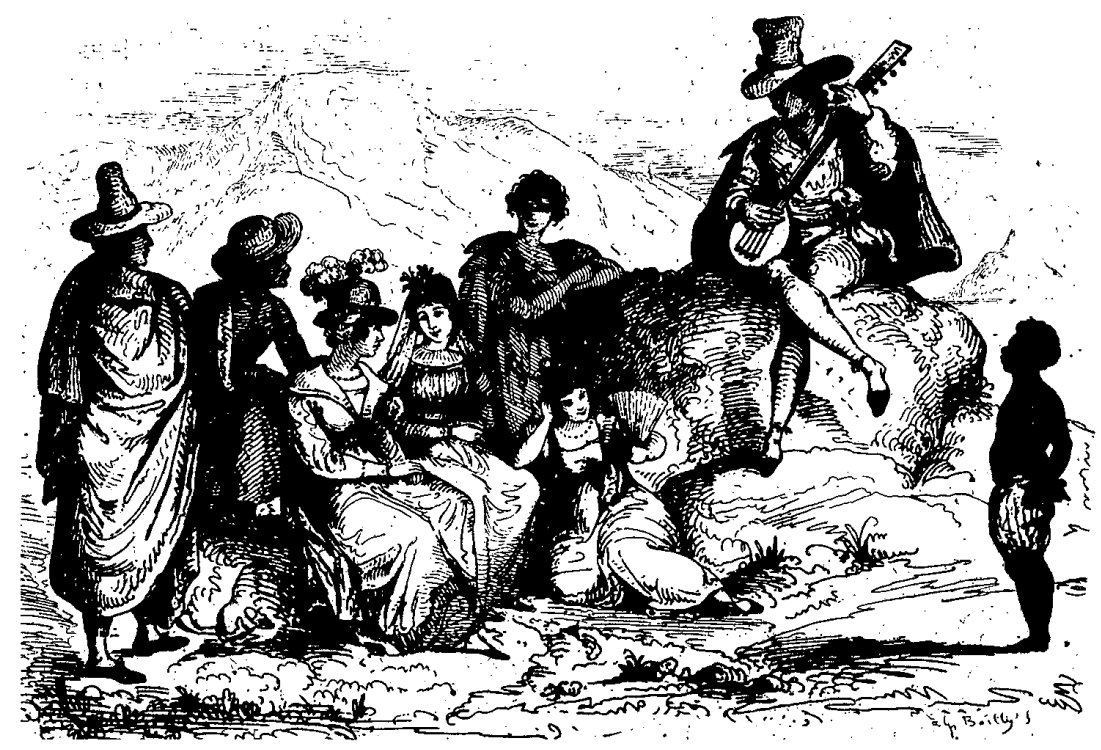

retomó la presidencia. Así, gran parte del electorado nunca había votado para elegir presidente, con seguridad sólo los mayores de 40 años habían tenido esa oportunidad.

Durante la dictadura militar, la elección del presidente de la república era indirecta, realizada por el Congreso que, en realidad, sancionaba la designación hecha por los militares. Este hecho diferencia a la dictadura brasileña de las demás del cono sur (Argentina, Chile y Uruguay), en las cuales ni siquiera existían los congresos, ni los partidos políticos.

La pretensión de los militares de mantener una vida republicana, aunque disminuida y maniatada, tuvo importantes consecuencias para la evolución posterior del sistema político, como veremos más adelante.

$\mathrm{Al}$ inicio de la dictadura, los militares intentaron mantener el sistema de parti- dos existentes antes del golpe; sin embargo, la derrota electoral del candidato oficial en las elecciones para gobernador del estado de Minas Gerais en 1965 , los llevó a suprimir los partidos y a sustituirlos por dos organizaciones nuevas. La Alianza Renovadora Nacional (ARENA) y el Movimiento Democrático Brasileño (MDB).

Los dos partidos se mantuvieron hasta 1979 cuando se volvió a reformar el sistema de partidos. Durante estos años las elecciones para legisladores (locales, estatales y federales), se realizaron con regularidad exceptuando los municipios considerados prioritarios para la seguridad nacional. El Congreso funcionó normalmente salvo un breve periodo en 1968 cuando fue cerrado por los militares.

Pese a sus errores y limitaciones, el funcionamiento del Congreso y la realización periódica de elecciones dotó a 
los partidos, artificialmente creados, de una función política y de representación en el régimen militar. Lo quisieran o no los militares, las elecciones representaban parte del proceso legitimatorio-deslegitimatorio del régimen y el Congreso era un contrapoder mínimo, pero que exigía sus prerrogativas y facultades.

El costo de ese juego político fue la destrucción real de los antiguos partidos políticos y su sustitución, sobre todo, por el MDB. Los procesos electorales habidos entre 1965 y 1978, fueron convirtiéndose en una grave preocupación para el régimen. Las elecciones se fueron convirtiendo en verdaderos plebiscitos de aprobación o rechazo al régimen, en los cuales el MDB se fortalecía como el frente opositor. ${ }^{1}$

El crecimiento del MDB y sus triunfos en 1978 pusieron en aprietos el proyecto de distensión política del presidente, general Geisel, llevándolo a reformar la legislación electoral y el sistema de partidos. Se puso fin al bipartidismo con la hipótesis de que el MDB se fraccionaría, debilitando a la oposición. De esta reforma nacieron el Partido do Movimiento Democrático Brasileiro (PMDB), el Partido dos Trabalhadores (PT), el Partido Trabalhista Brasileiro (PTB) y el Partido Democratico Trabalhista (PDT) como herederos del extinto MDB. De la ARENA surgió el Partido Democrático Social (PDS) y más tarde el Partido Popular (PP) y el Partido del Frente Liberal (PFL).

Pese a la reforma, el frente oposicionista, ahora aglutinado en el PMDB, conti-

\footnotetext{
${ }^{1}$ Considerando los votos para elegir senadores tenemos los siguientes porcentajes: en 1966 el MDB obtiene 34.2, en 1970, 28.6, en 1974, 50.0 yen 1978, 46.4. Para diputados federales los datos son los siguientes: 28.4 en 1966, 21.3 en 1970, 37.8en 1974 y 39.3 en 1978. Kinzo Gil, Maria da Alva, "Oposiçáo e autoritarismo. Génese e trajetoria do MDB, 19661979", IDESP-Vertice, Sao Paulo, 1988, p. 73.
}

nuó creciendo en las elecciones federales y para gobernadores hasta 1986 en que ganó 20 gubernaturas y la mayoría de los diputados federales, llevando a algunos observadores a afirmar que se estaba constituyendo un sistema si no de partido único, sí de partido predominante, lo cual podría ser desventajoso para la democracia y la alternancia en el poder.

Con todo, los analistas más atentos veían una dinámica diferente en la evolución del PMDB. Analizando los votos de las elecciones de la ciudad de Sao Paulo, cuyos datos presentamos en el cuadro 1 , se observa una declinación del partido en las últimas elecciones. Es posit le que los resultados muestren la vigencia del voto en contra o de descontento, pero ahora en contra del PMDB que ha gobernado el estado desde 1982, o bien que el electorado de la ciudad va definiendo mejor sus preferencias partidarias. Mas lo relevante para nosotros es la evidencia de que el triunfo del PMDB en las elecciones de 1986, en parte posibilitada por el momentáneo éxito del plan cruzado, difícilmente podían interpretarse como la emergencia de un sistema de partido dominante. Lo que se confirma a nivel nacional es el predominio del voto en contra del gobierno militar.

Entre 1986 y 1989 se dieron varios hechos importantes para entender los resultados de la elección presidencial.

El primero es el gran desprestigio del gobierno de Sarney, acusado de ineficiente y comupto, lo cual generó un fuerte desgaste del gobierno y de los partidos políticos que lo apoyaban, el PMDB y el PFL. Deslindarse de Sarney fue un objetivo central de todos los candidatos.

El segundo hecho importante fue el proceso de la Asamblea Constituyente. La elaboración de la nueva Constitución
1

8

1 


\author{
Cuadro 1 \\ VOTOS EMITIDOS EN FAVOR DEL PMDB PARA \\ PRESIDENTE MUNICIPAL Y GOBERNADOR EN LA CIUDAD \\ DE SAO PAULO DE 1982 A 1988
}

\begin{tabular}{lllll} 
Año & 1982 & 1985 & 1986 & 1988 \\
$\begin{array}{l}\text { Candidato } \\
\text { del PMDB }\end{array}$ & $\begin{array}{l}\text { Franco } \\
\text { Montoro } \\
\text { candidato a } \\
\text { gobernador }\end{array}$ & $\begin{array}{l}\text { Fernando } \\
\text { H. Cardoso } \\
\text { candidato a pte. } \\
\text { municipal }\end{array}$ & $\begin{array}{l}\text { O. Quercio } \\
\text { candidato a } \\
\text { gobernador }\end{array}$ & $\begin{array}{l}\text { Candidato del } \\
\text { PMDB a pte. } \\
\text { municipal }\end{array}$ \\
$\begin{array}{l}\text { Votos } \\
\text { obtenidos }\end{array}$ & $42.1 \%$ & $34.0 \%$ & $29.1 \%$ & $14.2 \%$ \\
$\begin{array}{l}\text { Resultado de } \\
\text { la elección }\end{array}$ & $\begin{array}{l}\text { Ganóla } \\
\text { elección }\end{array}$ & $\begin{array}{l}\text { Perdió la } \\
\text { presidencia } \\
\text { municipal } \\
\text { para Janio } \\
\text { Quadros (PTB) }\end{array}$ & $\begin{array}{l}\text { Perdió en la } \\
\text { capital para } \\
\text { Erminio de } \\
\text { Moras (PTB) }\end{array}$ & $\begin{array}{l}\text { Perdió para } \\
\text { Erondina } \\
\text { candidato } \\
\text { de (PT) }\end{array}$ \\
\hline
\end{tabular}

Fuente: Elaborado con datos publicados en Vinicius Caldeira Brant (coord.), Trabalhar e viver, Brasiliense, Sao Paulo, 1989, pp. 187 ss.

se demoró más de lo inicialmente planteado provocando sobrecarga informativa y aburriendo a los ciudadanos. Asimismo, el carácter derechista de algunos artículos disgustó a algunos sectores de la población, otros se sintieron defraudados por la permanencia del presidencialismo y por la excesiva injerencia del Estado en la economía y en la sociedad. En sintesis, el proceso de la constituyente acabó desgastando el tema de la política y de los partidos frente a la población.

El tercer hecho importante es la crisis de la economía y de la sociedad brasileña, manifestada en la hiperinflación, el descontrol de la política económica, el incremento de la violencia en las ciudades y en el campo, etcétera, que acabó por generar un amplio descontento en la población, independientemente de su nivel de ingresos.

Los tres factores señalados agudizaron el rechazo del electorado brasileño al gobierno, y no por ser militar sino por ser ineficiente y por la política desprestigiada como mecanismo de agregación y solución de los problemas generales. En síntesis y paradójicamente, el primer gobierno civil acabó profundizando el descontento de la población hacia lo político y hacia el gobierno. Las condiciones para el voto en contra eran propicias.

\section{LAS ELECCIONES, PRIMERA VUELTA}

En el cuadro 2, presentamos los resulr tados obtenidos por los 22 candidatos.

Aparte de indicar el triunfo de Collor y de Lula en la primera vuelta, lo que los facultó para participar en la segunda, los datos indican otros resultados interesantes. Los partidos más antiguos, el PDS, heredero de la ARENA, y el PMDB, del MDB, ocupan el $5^{\circ}$ y $7^{\circ}$ lugares respectiva- 


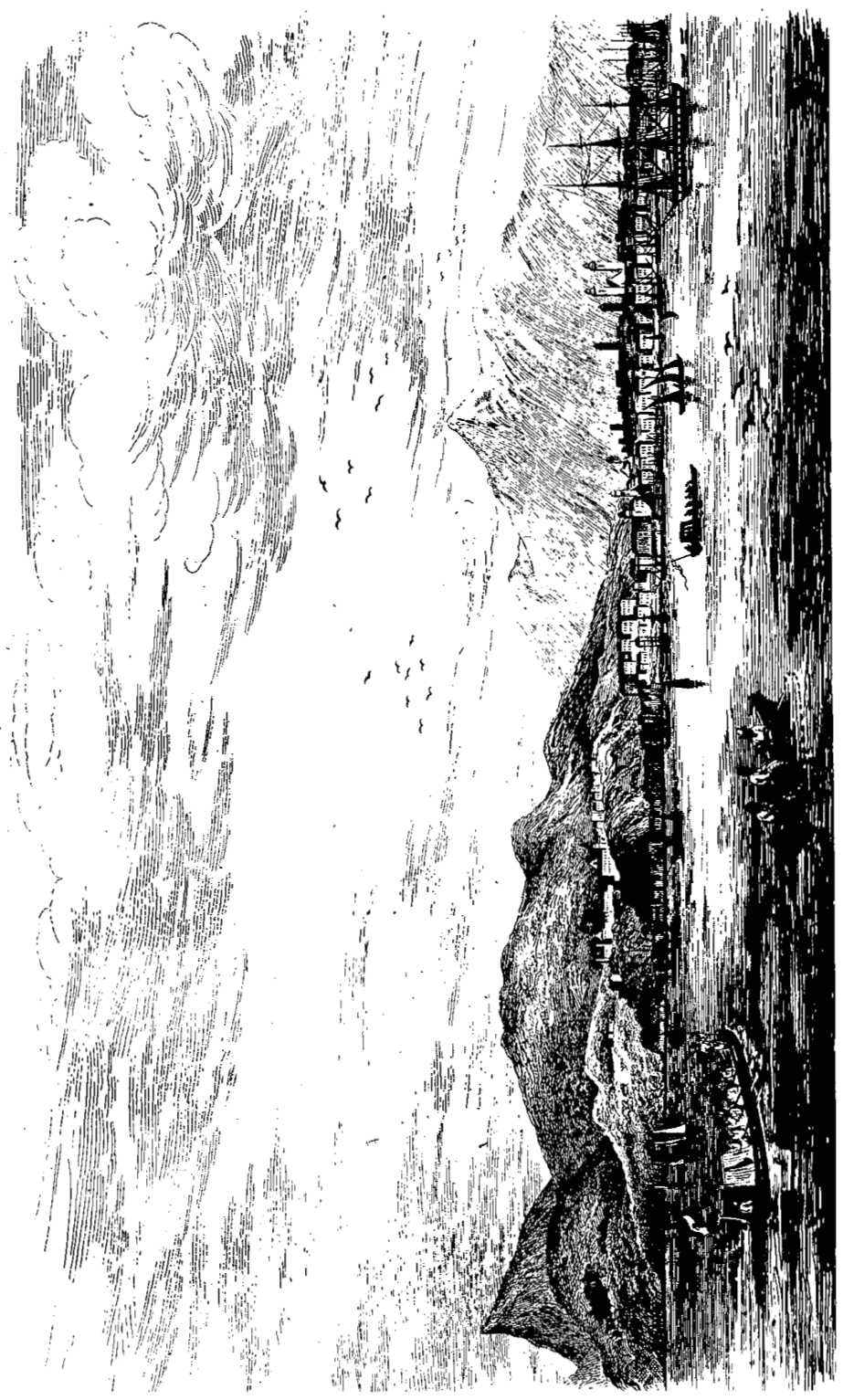


Cuadro 2

RESULTADOS DEL PRIMER TURNO DE LA ELECCIÓN POR

CANDIDATOS, NIVEL GENERAL

$\begin{array}{lcllrr}\begin{array}{l}\text { Fechade } \\ \text { creación }\end{array} & \begin{array}{c}\text { Coloca- } \\ \text { ción }\end{array} & \text { Nombre } & \begin{array}{c}\text { Sigla } \\ \text { opartido** }\end{array} & \begin{array}{c}\text { Total de } \\ \text { votos }\end{array} & \begin{array}{c}\text { Poncentaje } \\ \text { sobre el } \\ \text { total }\end{array} \\ \text { Partido Nuevo } & 1 & \text { Collor } & \text { PRN } & 20611011 & 28.52 \\ 1979 & 2 & \text { Lula } & \text { PI } & 11622673 & 16.08 \\ 1979 & 3 & \text { Brizola } & \text { PDI } & 11168228 & 15.45 \\ \text { Partido Nuevo } & 4 & \text { Covas } & \text { PSDB } & 7790392 & 10.78 \\ 1979 & 5 & \text { Maluf } & \text { PDS } & 5986575 & 8.28 \\ \text { Partido Nuevo } & 6 & \text { Afif } & \text { PL } & 3272462 & 4.53 \\ 1966-79 & 7 & \text { Ulysses } & \text { PMDB } & 3204932 & 4.43 \\ & 8 & \text { Freire } & \text { PL } & 769123 & 1.06 \\ 1979 & 9 & \text { Aureliano } & \text { PFL } & 600838 & 0.83 \\ \text { Partido Nuevo } & 10 & \text { Caiado } & \text { PSD } & 488846 & 0.68 \\ & 11 & \text { Camargo } & \text { PTB } & 379286 & 0.52 \\ & 12 & \text { Enéas } & & 360561 & 0.50 \\ & 13 & \text { Marronzinho } & & 238425 & 0.33 \\ & 14 & \text { PG } & & 198719 & 0.27 \\ & 15 & \text { Zamir } & & 187155 & 0.26 \\ & 16 & \text { Livia } & & 179922 & 0.25 \\ & 17 & \text { Mattar } & & 162350 & 0.22 \\ & 18 & \text { Gabeira } & \text { PV } & 125842 & 0.17 \\ & 19 & \text { Brant } & & 109909 & 0.15 \\ & 20 & \text { Pedreira } & & 86114 & 0.12 \\ & 21 & \text { Horta } & & 83286 & 0.12 \\ & 22 & \text { Correa* } & & 17363 & 0.01 \\ & & \text { Brancos } & & 1.43 \\ & & \text { Nulos } & & 3473484 & 4.23 \\ & & \text { Abstenciones } & & 82074718 & 100.00\end{array}$

- La candidatura fue impugnada por el Tribunal Superior Electoral (TSE). Esos votos fueron considerados nulos.

* Sólo anotamos las siglas de los partidos relevantes para nuestro análisis.

Fuente: Folba de Sao Paulo, 23 de noviembre de 1989.

mente; juntos no alcanzan $13 \%$ de la votación. En especial, es dramática la caída del PMDB y de su líder Ulysses Guimaraes, considerado en 1986 como el gran líder de la diretas ja y también líder de la Asamblea Constituyente.

Entre los cinco partidos que obtuvieron las más altas votaciones dos, el PRN de Collor y el PSDB de Covas, fueron creados después de 1979 (el segundo en 1988 y el primero en 1989) mostrando la enorme permeabilidad del sistema de partidos en Brasil y también el carácter tan volátil del votante.

Respecto a la votación por tendencias encontramos que los partidos de centro- 
Cuadro 3

RESULTADOS FINALES DE LA ELECCIÓN PARA PRESIDENTE DE LA REPÚBLICA EN EL TOTAL NACIONAL

$\begin{array}{lrc} & \text { Absolutos } & \text { Porcentajes } \\ \text { Collor } & 35089719 & 42.75 \\ \text { Lula } & 31076364 & 37.86 \\ \text { Blancos } & 986446 & 1.20 \\ \text { Nulos } & 3107893 & 3.79 \\ \text { Abstenciones } & 11814017 & 14.40 \\ \text { Total electores } & 82074718 & 100.00\end{array}$

Fuente: O estado de Sao Paulo, 31 de diciembre de 1989.

izquierda (PT, PDT, PSDB, PMDB, PCB, PTB Y pv) obtuvieron $48.49 \%$ del total de los votos emitidos, es decir hay una preferencia del electorado por los candidatos del centro-izquierda, y aunque el voto se encuentra muy disperso entre los partidos, sólo dos tienen más de $15 \%$. La dispersión en la derecha es peor, sobre todo si exceptuamos al PRN, que no era propiamente un partido, sino una sigla al servicio de un candidato, en este sentido el partido de derecha que más votos obtiene es el PDS, $8.28 \%$, y en conjunto retirando al PRN, sólo obtienen el 13.7 por ciento.

Los dos candidatos que ganaron el primer turno tienen características muy diferentes. En cuanto Lula era claramente un candidato clasista, obrerista, y presentaba un programa político radical (reforma agraria, moratoria al pago de la deuda externa, Estado benefactor, etc.) Collor era un populista de derecha que montó su campaña contra el gobierno de Sarney, contra la corrupción y contra los marajás que abusan de los puestos públicos haciendo promesas a los sectores populares. En general los candidatos claramente populistas, Collor, Brizola y Maluf, obtuvieron $52.25 \%$ de los votos, desmostrativos de una preferencia electoral de tipo tradicional, que privilegia al lider sobre el partido.
LAS ELECCIONES, SEGUNDA VUELTA

El triunfo inobjetable del candidato Fernando Collor de Mello esconde una gran cantidad de hechos, que es necesario desenrollar para entender el proceso en su conjunto.

En el cuadro 4 reproducimos las votaciones obtenidas por Lula en cada una de las ciudades capitales y de cada estado para poder comprender el significado de la distribución. Hemos preferido hacer el cuadro con la votación otorgada a Lula por representar a un electorado más consistente y explicable, mientras que el voto a Collor aparece muy confuso.

De acuerdo con los datos, Lula ganó en las mayores ciudades, con la excepción de Sao Paulo; considerando las diez mayores, Lula obtuvo más votos que Collor en siete ciudades. De la misma manera, Lula triunfó en dos de los tres estados más importantes. Estos datos indican que Lula contó con la preferencia de la mayoría de los electores más bien urbanos, en tanto que Collor fue favorecido por los del interior del país y en especial, por aquellos que viven en los estados más pobres.

La información muestra también que los triunfos que ganó Lula en las ciudades disminuyen gradualmente en el 
Cuadro 4

VOTOS OBTENIDOS POR LULA EN LOS ESTADOS Y CAPITALES

\begin{tabular}{|c|c|c|c|}
\hline Nombres & $\begin{array}{l}\text { Porcentaje } \\
\text { ciudad }\end{array}$ & $\begin{array}{c}\text { Porcentaje } \\
\text { estado }\end{array}$ & $\begin{array}{l}\text { Diferencia } \\
\text { en porcentaje }\end{array}$ \\
\hline Sao Paulo, Sao Paulo & 37.40 & 36.43 & 0.97 \\
\hline $\begin{array}{l}\text { Río de Janeiro, } \\
\text { Río de Janeiro }\end{array}$ & 6463 & 6379 & 0.82 \\
\hline Belo Horizonte, & 0.03 & 05.17 & 0.02 \\
\hline Minas Gerais & 56.67 & 35.57 & 21.10 \\
\hline Vitoria, Espíritu Santo & 41.27 & 33.64 & 7.63 \\
\hline Porto Alegre, RGS & 66.46 & 59.06 & 7.4 \\
\hline Florianópolis, & & & \\
\hline Sta. Catarina & 58.88 & 42.23 & 16.65 \\
\hline Campo Grande, & & & \\
\hline Mt. Grosso do Sul & 29.90 & 26.91 & 2.99 \\
\hline Brasilia, D.F. & 52.70 & 52.70 & 0 \\
\hline Miragema do & & & \\
\hline Tocantins,Tocantins & 31.17 & 13.48 & 17.69 \\
\hline Rio Blanco, Acre & 27.62 & 21.72 & 5.9 \\
\hline Belem do Pará, Pará & 34.42 & 19.19 & 15.23 \\
\hline Macapá, Amapa & 29.01 & 25.33 & 3.68 \\
\hline Aracaju, Sergipe & 41.84 & 26.91 & 14.93 \\
\hline Natal, RGN & 52.51 & 37.17 & 15.34 \\
\hline Joao Pessoa, Paroiba & 47.16 & 34.53 & 12.63 \\
\hline Curitiba, Parano & 37.25 & 26.91 & 10.34 \\
\hline Goiania, Goiais & 40.17 & 24.33 & 15.84 \\
\hline Cuiaba, Mato Grosso & 37.92 & 23.39 & 14.39 \\
\hline Porto Velho, Rondonia & 30.10 & 21.54 & 8.56 \\
\hline Boa Vista, Roraima & 17.96 & 16.94 & 1.02 \\
\hline Manaus, Amazonas & 30.75 & 23.45 & 2.30 \\
\hline Maceio, Alagoas & 28.68 & 17.75 & 10.93 \\
\hline Recife, Pernambuco & 57.19 & 40.09 & 17.07 \\
\hline Salvador, Bahía & 60.58 & 33.61 & 26.97 \\
\hline Sao Luis, Maranhao & 43.18 & 24.34 & 18.67 \\
\hline Terezinha, Piauí & 49.53 & 30.86 & 18.67 \\
\hline Fortaleza, Ceará & 52.07 & 33.40 & 18.67 \\
\hline
\end{tabular}

Fuente: O Estado de Sao Paulo, 31 de diciembre de 1989

interior de cada estado, salvo en Río de Janeiro, Río Grande del Sur y Distrito Federal, con lo cual se reafirma el carácter rural, interioreño, del rechazo a Lula.

En el cuadro 5 hemos agrupado las votaciones obtenidas por los candidatos en los cinco y diez estados más impor- tantes, en los 17 restantes y en las cinco y diez ciudades más importantes.

La información agregada muestra con toda claridad la relación entre el voto a Lula y la urbanización y grado de desarrollo del estado o ciudad. El voto populista dado a Collor fue de origen funda- 
Cuadro 5

VOTACIONES DENEGADAS POR ESTADO Y CIUDADES.

DATOS DEL SEGUNDO TURNO

\begin{tabular}{|c|c|c|c|c|c|c|}
\hline & $\begin{array}{c}\text { Votaciön } \\
\text { total }\end{array}$ & $\begin{array}{c}\text { Votación } 5 \\
\text { estados } \\
\text { mayores }\end{array}$ & $\begin{array}{c}\text { Votación } 10 \\
\text { estados } \\
\text { mayores }\end{array}$ & $\begin{array}{c}\text { Votación } 17 \\
\text { estados } \\
\text { menores }\end{array}$ & $\begin{array}{c}\text { Votación } 5 \\
\text { ciudades } \\
\text { mayores }\end{array}$ & $\begin{array}{c}\text { Votación } 10 \\
\text { ciudades } \\
\text { menores }\end{array}$ \\
\hline Collor & 35089998 & 19049890 & 27469505 & 7620493 & 4630809 & 5943148 \\
\hline Lula & 31076364 & 20670473 & 28444531 & 2631833 & 6450902 & 8383007 \\
\hline d/abs." & +4013634 & -1620583 & -975026 & +4988660 & -1820093 & -2439859 \\
\hline
\end{tabular}

mentalmente rural y en los estados más atrasados. Sólo el estado y la ciudad de Sao Paulo muestran un comportamiento diferente pareciendo negar la relación entre urbanización y voto por Lula. En el caso de Sao Paulo se pueden plantear dos hipótesis alternativas. La primera apunta a que el comportamiento del electorado paulista muestra una definición partidaria mucho mayor que los otros estados, el pluripartidismo explicaría la especificidad; esta hipótesis tendría como contraparte que en Río de Janeiro y Río Grande del Sur, donde ganó Lula, resultó determinante el liderazgo de Brizola, quedando Brasilia, D.F., como excepción, dado que ahí hay un claro predominio petista. Recuérdese que fue el único estado donde Lula ganó en la primera vuelta. La segunda hipótesis diría que la derrota de Lula en Sao Paulo se pudo deber a que sufrió el efecto del voto en contra de las administraciones petistas en municipios como Sao Paulo o Santos o en la región del ABC. Infelizmente no disponemos de información desagregada para confirmar o negar esta hipótesis. El caso de Porto Alegre en donde también administra el municipio el PT, el voto en contra, quizás mostrado en la primera vuelta cuando Lula obtuvo una votación bajísima, fue anulado en la segunda por la influencia de Brizola.

En todo caso, lo que sí podemos constatar es que los votos que obtuvieron los partidos de centro e izquierda no fueron transferidos en su totalidad a Lula, en tanto que Collor sí contó con la votación de los partidos de derecha. Los datos aparecen en el cuadro 6.

De acuerdo con las cifras se puede afirmar que Lula no consiguió recuperar todos los votos que fueron otorgados a los partidos de centro-izquierda en la primera vuelta. Se puede presumir que los votos no transferidos pertenecen a los electores de centro del brizolismo en Río de Janeiro o Río Grande del Sur, o bien del PSDB o del PMDB en Sao Paulo y Minas Gerais. Estos casos mostrarían también la existencia de un electorado de izquierda más consistente, el cual votó por Lula en el segundo turno.

La hipótesis de que los votos que Lula perdió fueron transferidos a la candidatura de Collor no es necesariamente cierta. Restando los votos otorgados a partidos de izquierda del total de los votos emitidos a favor de los dos candidatos, es decir, sin considerar los votos blancos o nulos ni las abstenciones, tenemos. 32573539 votos de centro-izquierda, 
Collor obtiene en la segunda vuelta 35089998 , dando una diferencia de 2516459 votos, casi 1500000 menos de los no transferidos al nivel total de Brasil. Así mismo, vemos que además aumentaron en poco más de 2000000 las abstenciones y los votos nulos, y que los votos en blanco disminuyeron cerca de 450000 votos, lo cual casi anula la diferencia obtenida por Collor en la segunda vuelta con respecto a la obtenida por los partidos que no son de centroizquierda. En todo caso la hipótesis necesitaría de datos más finos para comprobarse. Aquí sólo descartamos la relación directa entre votos de centro izquierda no transferidos a Lula y otorgados a Collor.

En términos numéricos podemos afirmar que Collor ganó con el apoyo de los ciudadanos del interior del Brasil, en especial de los estados y ciudades más pobres y paradójicamente con los votos del estado más rico tanto de su capital como del interior: Sao Paulo. En cambio Lula perdió por lo mismo, más por la desconfianza de parte del electorado de centro izquierda que le negó su voto. En este comportamiento pudo influir el carácter clasista del programa de Lula, sobre lo cual insistió mucho el PSDB, y también debió influir la campaña agresiva y de descalificación moral de Collor sobre la personalidad de Lula.

\section{LAS ELECCIONES Y LA TRANSICIÓN POLITICA EN BRASIL}

La información presentada y la interpretación que hemos hecho de ella nos permite destacar dos hechos fundamentales. El primero es la fragilidad del sistema de partidos, y el segundo se refiere al comportamiento del electorado, es decir sus amplios desplazamientos, su

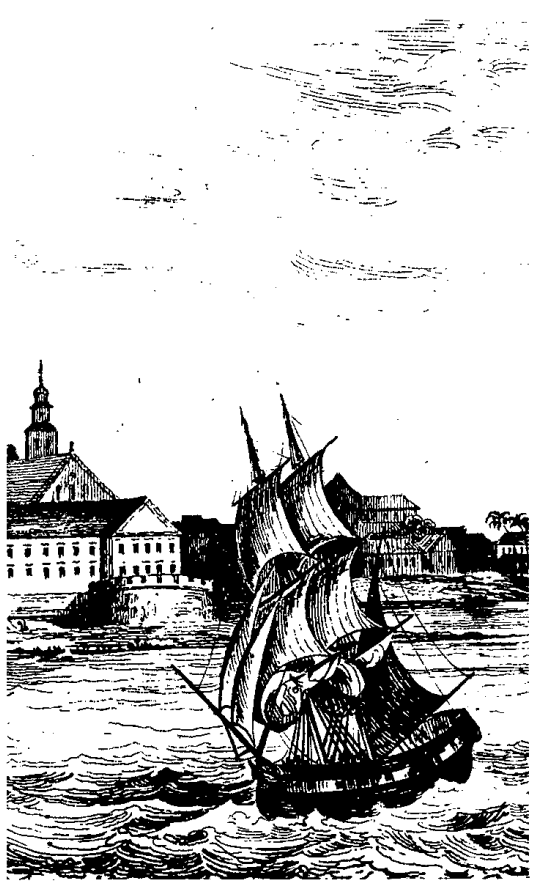

baja consistencia o predictibilidad. Ambos hechos están estrechamente relacionados con la transición política.

La falta de un sistema de partidos más o menos estable y de fidelidades partidarias de los electores es un problema serio para la consolidación de un sistema político democrático e introduce incertidumbres en la transición.

El swing del electorado muestra dos hechos preocupantes. Por una parte, la preferencia por candidatos de tipo populista, sean de izquierda o de derecha, que se expresa como adhesión y fidelidad a un candidato y no a un partido, muestra simpatía por un discurso político mucho más emotivo que racional y que permite rápidas y fugaces identificaciones. Por la otra, una cultura política del voto en 
Cuadro 6

COMPARACIÓN DE LOS VOTOS OBTENIDOS POR LOS PARTIDOS DE IZQUIERDA EN EL PRIMER TURNO Y LOS OBTENIDOS POR LULA EN EL SEGUNDO TURNO, EN BRASIL Y ESTADOS ESCOGIDOS

\begin{tabular}{|c|c|c|c|c|c|}
\hline & Brasil & $\begin{array}{l}\text { Río de } \\
\text { Janeiro }\end{array}$ & $\begin{array}{c}\text { Rio Grande } \\
\text { del Sur }\end{array}$ & $\begin{array}{l}\text { Mina } \\
\text { Gerais }\end{array}$ & Sao Paulo \\
\hline \multicolumn{2}{|c|}{$\begin{array}{l}\text { Votos obtenidos } \\
\text { por los partidos } 35057473 \\
\text { de izquierda en la } \\
\text { primera vuelta }\end{array}$} & 5682729 & 4093437 & 3569966 & 7469221 \\
\hline $\begin{array}{l}\text { Votos obtenidos } \\
\text { por Lula en la } \\
\text { segunda vuelta }\end{array}$ & 31078364 & 5228265 & 3366802 & 3355121 & 6739378 \\
\hline $\begin{array}{l}\text { Diferencia } \\
\text { de votos }\end{array}$ & 3984109 & 454646 & 726635 & 214845 & 728843 \\
\hline $\begin{array}{l}\% \text { sobre los } \\
\text { obtenidos } \\
\text { en la primera } \\
\text { vuelta }\end{array}$ & $-11.36 \%$ & $-8.00 \%$ & $-18.00 \%$ & $-6.00 \%$ & $-9.75 \%$ \\
\hline
\end{tabular}

Fuente: Elaborada en base a los datos de la primera vuelta publicados por $A$ Folba de Sao Paulo, y los de la segunda vuelta publicados en O Estado de Sao Paulo.

contra, de reprobación del régimen, que continúa dando a las elecciones un cierto carácter plebiscitario. Desde luego no estamos criticando el voto en contra, lo que parece negativo es que la acción ciudadana no contemple su voto de manera también positiva, racional, definiendo una opción futura.

La combinación del voto en contra y de la cultura populista da al sistema político una inestabilidad que puede ser limitante para las instituciones partidarias. La elección de Collor es una muestra de ello: con una sigla partidaria de membrete, con una campaña política montada sobre un esquema populista, logra desplazar a los partidos políticos constituidos y con trabajo permanente.

Visto desde el enfoque del sistema de partidos, el problema es también grave. El swing del electorado hace inconsistente el sistema de partidos y sus relaciones, crea mayorías o minorías poco previsibles y por tanto dificulta las ya de por sí críticas formas de representación de la ciudadanía. El hecho de que el mayor partido hasta antes de la elección, con gran mayoría en el Congreso, desaparezca prácticamente del panorama electoral es tan insólito como grave en un sistema político constituido.

Los hechos señalados no son nuevos en el sistema político brasileño, pero dado que ahora se manifiestan en la primera elección presidencial de los últimos 25 años, cobran importancia y dan señales de alerta para evitar dificultades en la construción de la democracia. 


\section{SECUENCIA}

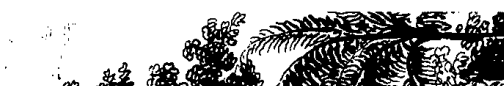

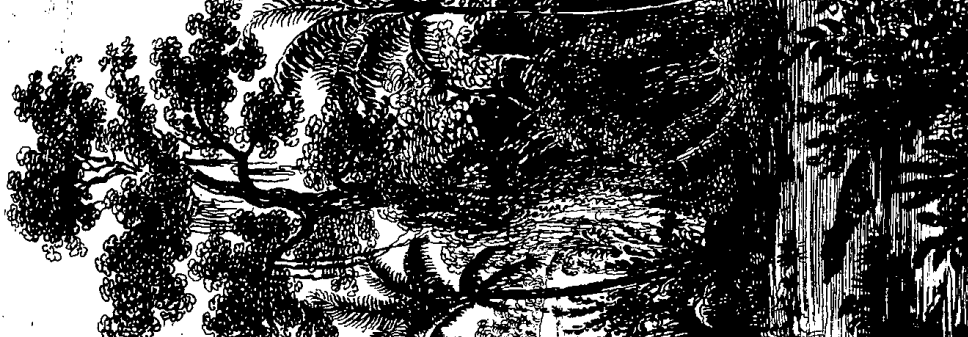

(20)

of

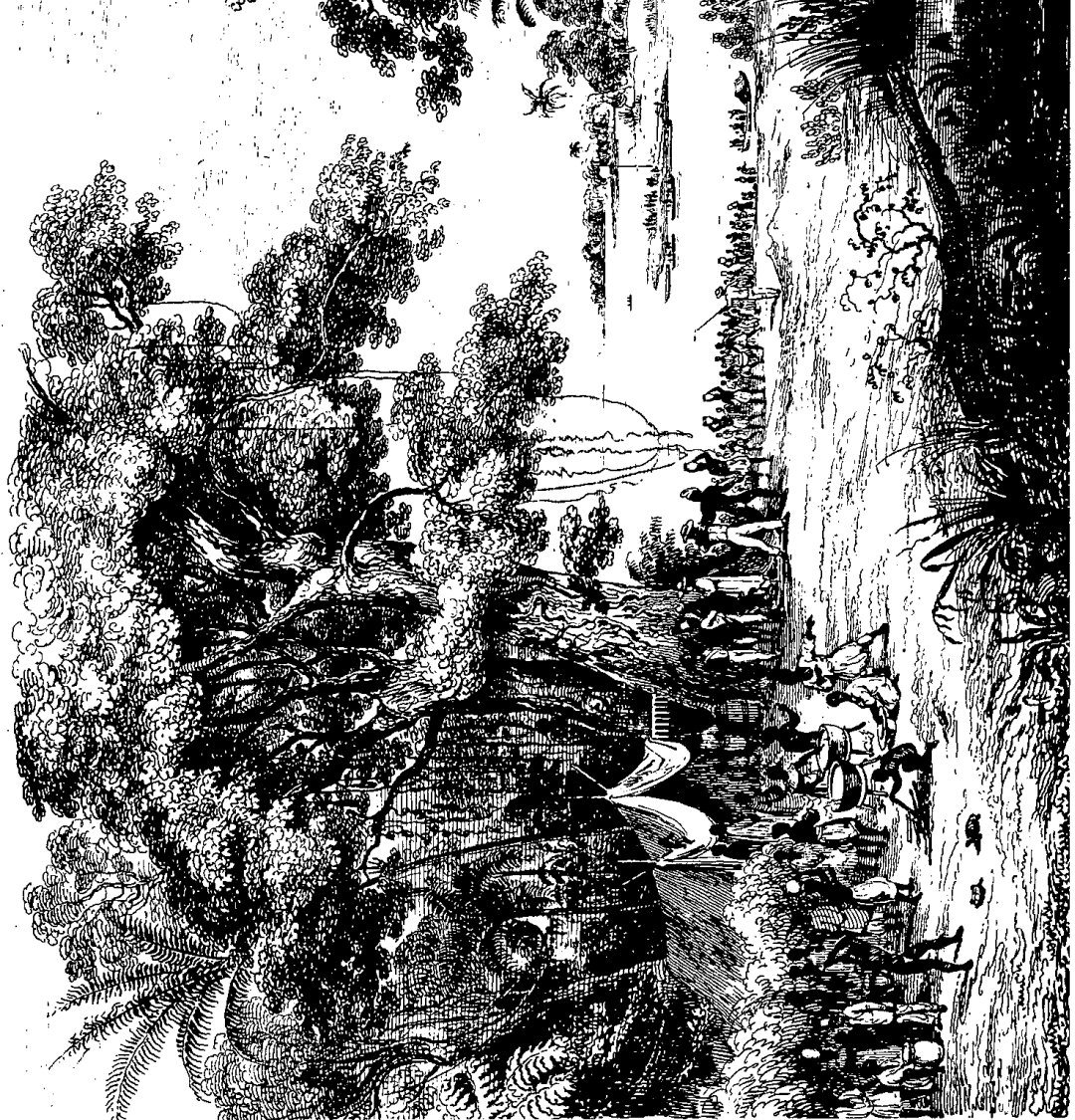

